\title{
"In-situ microscopy for real-time determination of single- cell size distribution and activity in microbial cultures"
}

\author{
A. Marbà-Ardébol ${ }^{1}$, J. Emmerich ${ }^{2}$, P.Neubauer ${ }^{1}$, S.Junne ${ }^{1}$ \\ ${ }^{1}$ Chair of Bioprocess Engineering Department of Biotechnology, Technische Universität Berlin \\ Ackerstrasse 76, ACK 24, D-13355 Berlin, Germany \\ 2 SOPAT GmbH, Boyenstr. 41, D-10115 Berlin, Germany \\ a.marbaardebol@tu-berlin.de
}

\begin{abstract}
Summary
A photo-optical in-situ microscopy device was adapted and applied to monitor morphological features of single cells directly in the cell suspension of bioreactors. The real-time measurement is conducted by coupling of the photo-optical probe to an automated image analysis.

Several parameters, which are gained from the captures of the microscope, can be related to process relevant features like growth, cell activity and lipid content in algae and yeast cultivations. Moreover, the single-cell distribution of several morphological parameters provides an information about the cell activity, and the degree of population homogeneity.

The possibility to monitor changes in cell morphology directly on-line enables to gain new parameters for monitoring and control on a single-cell level, in process development as well as in production scale.
\end{abstract}

Keywords: in-situ, monitoring, cell size, algae, budding.

\section{Introduction}

The generation of data in real-time allows a better way of process monitoring, development and optimization, especially in the era of automation and digitalization. So far, only conventional parameters such as $\mathrm{pH}$-value, dissolved oxygen concentration or the exhaust gas composition are frequently measured on line in bioprocesses. This means that neither physiology of the cells nor their heterogeneity is considered, while total biomass and viability measurements are conducted in suspension rarely and only on a volumetric basis.

However, advances in in situ microscopy also allow the on line monitoring of the single-cell size distribution in order to characterize the morphological cell state. Furthermore, the physiological state is often related to the morphological state, so there is a relationship between form and function.

The morphology is not only altered by the process conditions, but each cell is influenced by the cell cycle, the aging, or the lifeline along the reactor. Therefore, the morphology is a dynamic and heterogenic parameter.

Consequently, in order to quantify and detect this heterogeneity, data on a single-cell level are needed. Moreover, in order to establish valid relations between cell physiology and morphology, a statistically representative sample of the population has to be measured. This becomes critical for off line and at line measurements, since sampling, and usually sample preparation is required.

Therefore, the photo-optical in-situ microscopy (ISM) used in the present study, based on the determination of morphological parameters on the single-cell level, constitutes a meaningful analytical tool, which gains appropriate data to assess the population status in the cultivations. This ISM consists of a single-rod sensor probe that allows the capture of images within a known focus area, in an adjustable measurement gap, using a high-resolution CCD sensor. Cells continuously pass through this gap due to the movement of the liquid phase. The flash light illumination is conducted by transmission, therefore the light originates from the opposite side of the camera [1]. The probe can be installed directly in the bioreactor and dipped into the cell suspension, or it can be used through a by-pass line.

The microscopy analysis can be split into three steps: (i) image acquisition, (ii) particleidentification and (iii) data-analysis. All steps need to be adapted to the organism of the study. 
The magnification or the stroboscope intensity can be adjusted. Particle-identification is performed by training of the software for segmentation and classification of cells. Moreover, the automatic identification and evaluation circumvent biased results usually obtained by off line manually human based analysis.

Until now, two selected microorganisms, namely the heterotrophic microalgae Crypthecodinium cohnii and the yeast Saccharomyces cerevisiae, have been investigated.

\section{Case study 1:}

As an example of the effect of an industrially relevant stress factor, the accumulation of the polyunsaturated fatty acid docosahexaenoic acid (DHA) in the heterotrophic microalgae $C$. cohnii by nitrogen limitation was investigated.

DHA is a fish feed substitute for aquaculture (DHA is so far obtained from fish oil). Hence, biotechnologically produced DHA represents a sustainable alternative to replace fish oil and fish meal production.

The process can be divided into two parts. The growth phase, in which the cells divide and become smaller, and the production phase, in which the cells accumulate the product, and thus become larger (Fig. 1).

Besides ISM, digital holographic microscopy (DHM) was used as an at-line reference method. The cell size measured with ISM and DHM was used to determine the process state, in which growth and DHA production is favorable.

Finally, it could be demonstrated that the accumulation of the product can be quantitatively detected by means of ISM (Fig. 2) [2].

The method is currently used to investigate the impact of shear forces on the morphological heterogeneity.
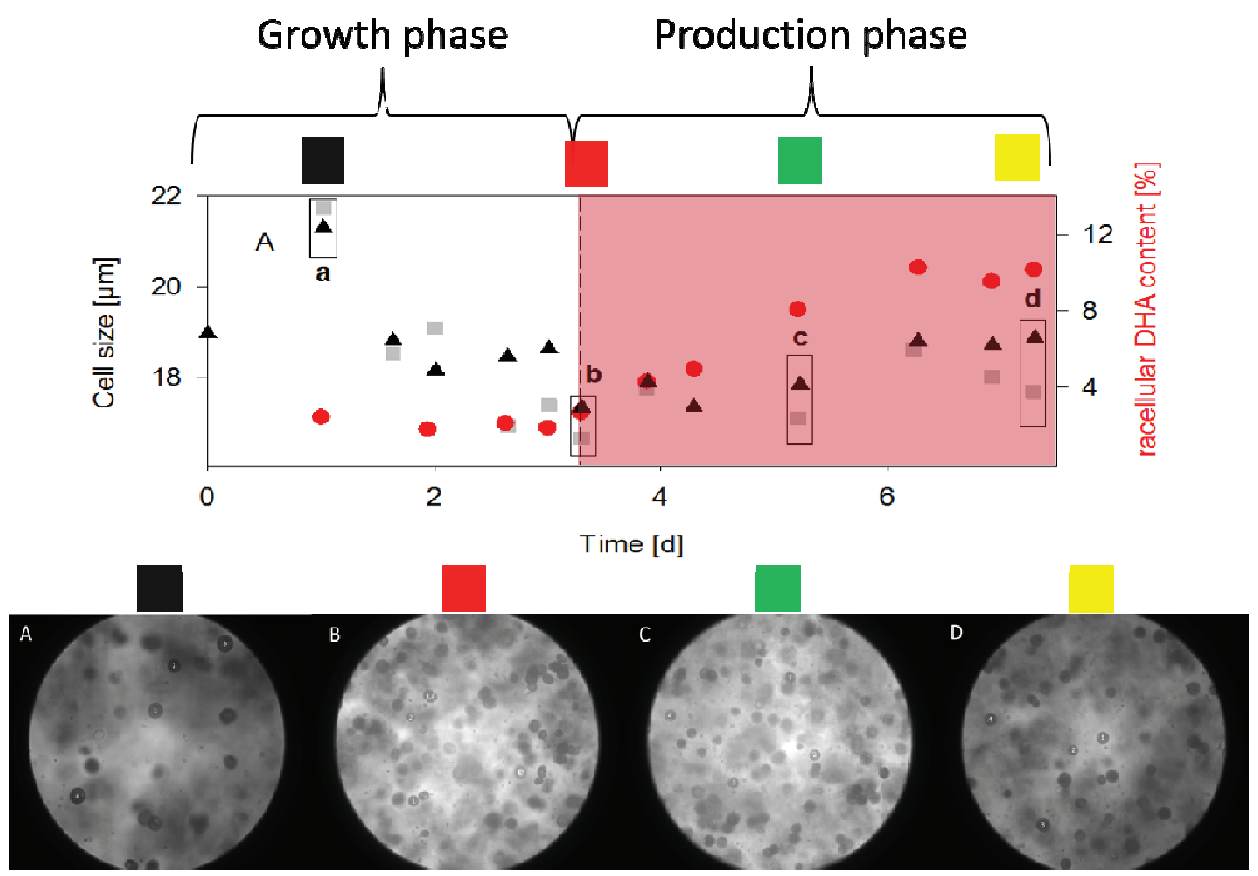

Fig. 1: $\quad$ The diagram shows the measured cell size variation with ISM (triangle) and DHM (square) caused by the intracellular product accumulation of DHA (circle). Bottom line: pictures taken at four times during the first day (A), after 2.9 days (B), after 5.2 days (C) and after 7.3 days (D) of cultivation. 


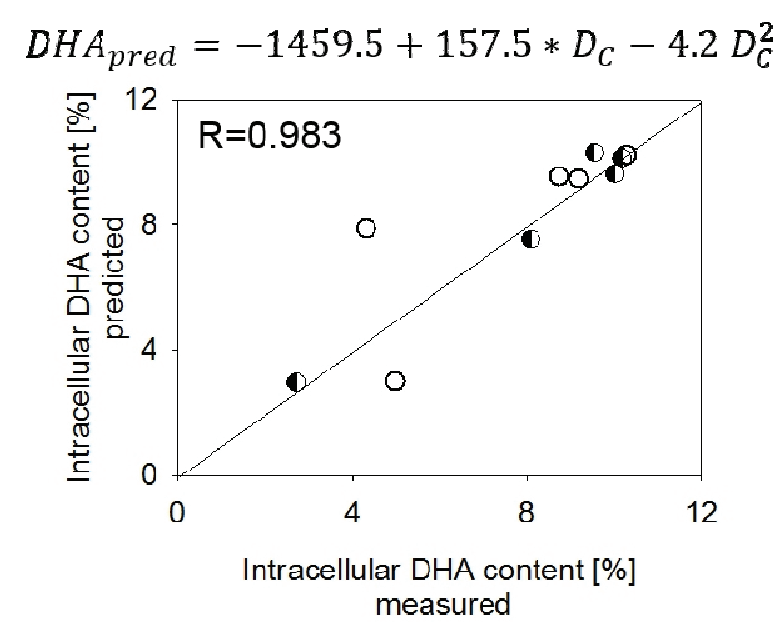

Fig. 2: Second-order correlation between DHA content measured off line (using gas phase chromatograph equipped with a flame ionization detector) and predicted using the average $d_{32}$ as detected on line by ISM and crosscalibration. Values used for calibration (0) and prediction (0).

\section{Case study 2:}

With a further development of the probe, the maturation state of the budding yeast $S$. cerevisiae was quantified. In the case of the budding yeast, the proportion of cells that are in the maturation state at a time (budding index, $\mathrm{BI}$ ), provide information of the growth status. Moreover, their viability was defined as the cell's ability to bud and grow.

The automatic cell recognition was able to identify and distinguish budding and nonbudding (or daughter) cells successfully in cell suspension if compared to manual counting (Fig. 3). Based on this classification, the budding index was automatically calculated. In order to ensure that a representative sample of the cell population was measured, a sensitivity analysis from each cell class (budding and non-budding) was performed, since both classes present a different grade of heterogeneity. The different degree in heterogeneity is in part due to the segmentation criteria adopted. The coefficient of variance of $\mathrm{BI}$, when measuring the same sample three times independently, was less than $0.15 \%$. Shadow of effects for false negative results, budding occurs in the optical shadow of a cell) can be neglected, as it accounts to less than $2 \%$ of recognized cells.
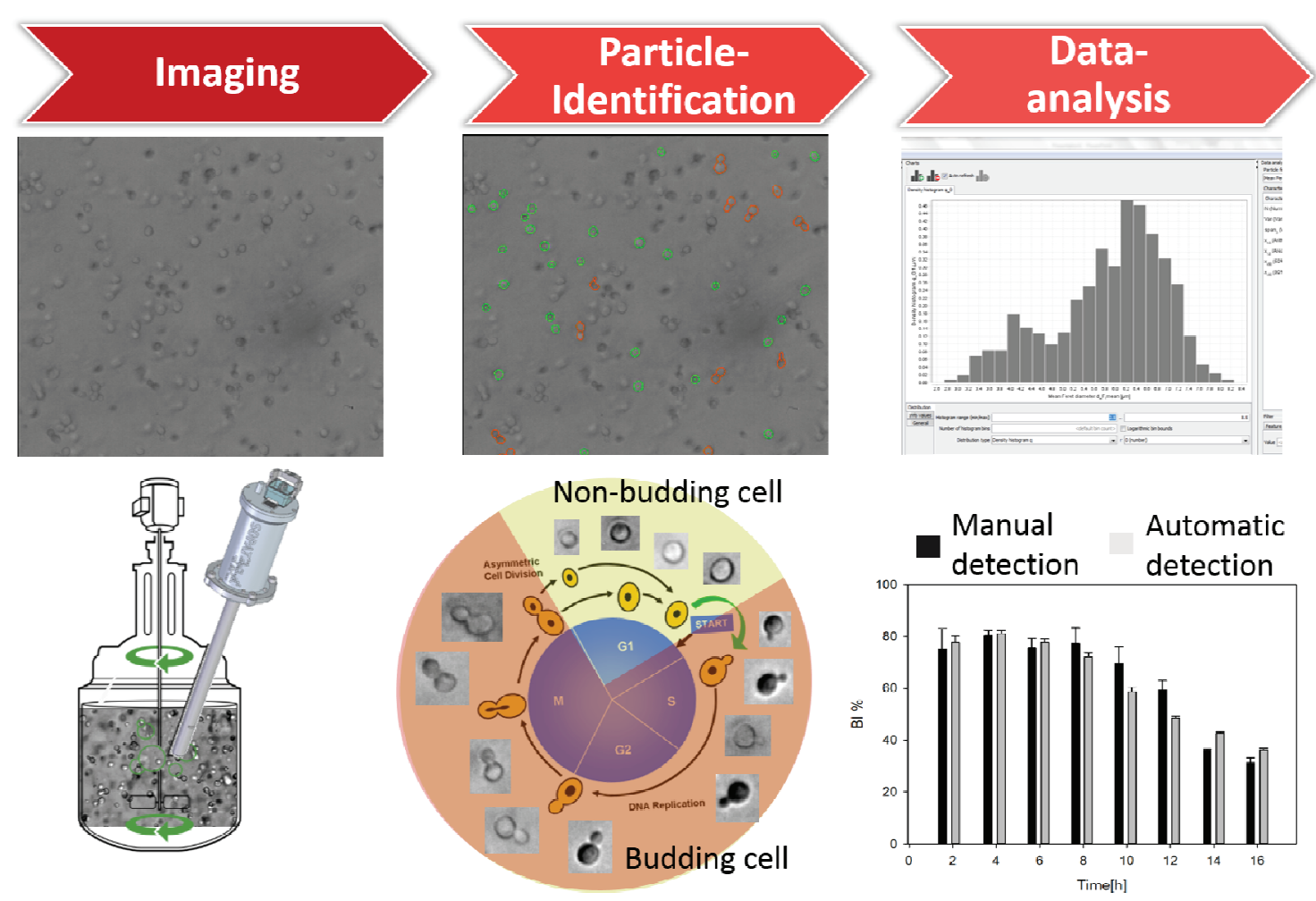

Fig. 3: Workflow used by the ISM. The classification of the cells as determined by automatic particle analysis: non-budding (G1) and budding (S, G2 and M). Besides, the comparison between the Bl obtained through automatic image recognition and manual classification is shown. 
The single-cell size distribution of several morphologic parameters, namely the cell diameter, the circularity and aspect ratio, is obtained for each class. Thus, conditions providing an active growth state can be identified on-line for the first time. Moreover, the single-cell distribution provides not only information about the cell activity, but also about the population heterogeneity.

\section{Acknowledgements}

This research is conducted with support of the German Federal Ministry of Economics and Energy within the framework program ZIM-Koop, project "Smart Process Inspection", grant no. ZF 4184201CR5.

\section{Literature:}

1. Panckow, R.P., et al., Photo-Optical In-Situ Measurement of Drop Size Distributions: Applications in Research and Industry. Oil Gas Sci. Technol. - Rev. IFP Energies nouvelles, 2017. 72(3): p. 14.

2. Marbà-Ardébol, A.-M., et al., Single-cellbased monitoring of fatty acid accumulation in Crypthecodinium cohnii with three-dimensional holographic and in situ microscopy. Process Biochemistry, 2017. 52: p. 223-232. 